\title{
Clinical Research on Tooth Extraction under Acupuncture Anesthesia
}

\author{
GU Zu-qian (幸祖谦), WANG Yan-qiu (王燕秋), YIN Xiao-rong（殷蓉蓉), \\ YIN Ye-xiang (尹业祥), YU Ming-hui (余明惠), \\ WANG Hua-jun (王华均), CHEN Wei-min (陈卫民) \\ Department of Stomatology, Tongii Medical University, Wuhan
}

This paper is a résume of the results of clinical research on tooth extractions under acupuncture anesthesia (AA) during the 10 year period from March 1972 to Feb. 1983 in our department, with the number totalling 3150 cases. There were altogether 3691 permanent teeth extracted.

The ma:ority of patients were stimulated by electric current with acupuncture anesthesia apparatus (AAA) and the five acupoints in the maxillofacial region most commonly used were Renkuang(人眶), Chenghai(承额), Bichungou(算唇沟), Yao.ishang(㕮肌上) and Xiachicao(下 齿槽).

The effects of AA were divided into 4 grades. The rates of grades I and I varied from $60.01 \%$ to $86.73 \%$ in all cases, with an average of $80.12 \%$.

A statistical analysis of the main data is given below.

The effect of tooth extractions under AA appeared to bear no relation to sex $(P>0.05)$. Nor does it bear any relation to the duration of operation $(P>0.05)$. Regarding its relation to the induction time, no significant difference was observed in all groups of patients ( $P>$ 0.05). Usually, 7-14 min were used.

However, the effects of tooth extraction under AA prove to be related to the following factors.

Age: The effects of AA were worse in patients under 15 than in any other age groups. $(P<0.01)$.

The position of teeth: The effects of AA were better in the maxillary teeth than in the mandibular ones, and also so in the anterior ones than in the rosterior ones, with the best effects in anterior maxillary teeth, and the worst in lower molars $(P<0.01)$.

The acupoints: They were relatively specific. Significant difference was observed in different acupoints, with the worst effect in Xiachicao $(P<0.01)$.

Type of diseases: Tooth extractions for prosthesis and orthodontics, etc, resronded best to AA, while the impacted teeth and tooth extractions in an acute inflammatory state responded most poorly $(\mathrm{P}<0.01)$.

In our opinion, AA for tooth extractions possesses many advantages: It is safe, convenient for use, and free from complications or allergic reactions. In addition, the postoperative reactions are mild. As a result, the majority of patients need no sedatives.

It was also found that the intensity to electrical stimulation was stable and the frequency equal, adjustable, persistent and easy to control. Better AAA can even raise the rates of grades $I$ and $\mathbf{I}$.

From the above it may be concluded that this method can be used more widely except for imfacted teeth, tooth extraction in acute inflammatory state and non-vital teeth, in all of which, however, AA can still be used selectively.

(A color film ( $16 \mathrm{~mm}, 9 \mathrm{~min}$ ) is available from the authors) 